\title{
Symposium: Sex Differences in Musculoskeletal Disease and Science
}

\section{Editorial Comment: Symposium: Sex Differences in Musculoskeletal Disease and Science}

\author{
Karl J. Jepsen PhD, Nancy E. Lane MD, John H. Healey MD, \\ Laura L. Tosi MD
}

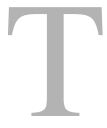
he US Public Health Service noted in 1985 that lack of knowledge about female biology compromises the health of women. But until the 1990s, biomedical research was primarily rooted in the male model - the belief that male biology (outside of the reproductive system) was representative of the

The authors certify that they, or any members of their immediate families, have no funding or commercial associations (eg, consultancies, stock ownership, equity interest, patent/licensing arrangements, etc.) that might pose a conflict of interest in connection with the submitted article. All ICMJE Conflict of Interest Forms for authors and Clinical Orthopaedics and Related Research ${ }^{\circledR}$ editors and board members are on file with the publication and can be viewed on request.

The opinions expressed are those of the writers, and do not reflect the opinion or policy of $C O R R^{\circledR}$ or the Association of Bone and Joint Surgeons ${ }^{\circledR}$.

\section{K. J. Jepsen $\mathrm{PhD}$}

Department of Orthopedic Surgery,

University of Michigan, Ann Arbor, MI, USA

N. E. Lane MD

Division of Rheumatology, Department of Medicine, University of California-

San Francisco, San Francisco, CA, USA

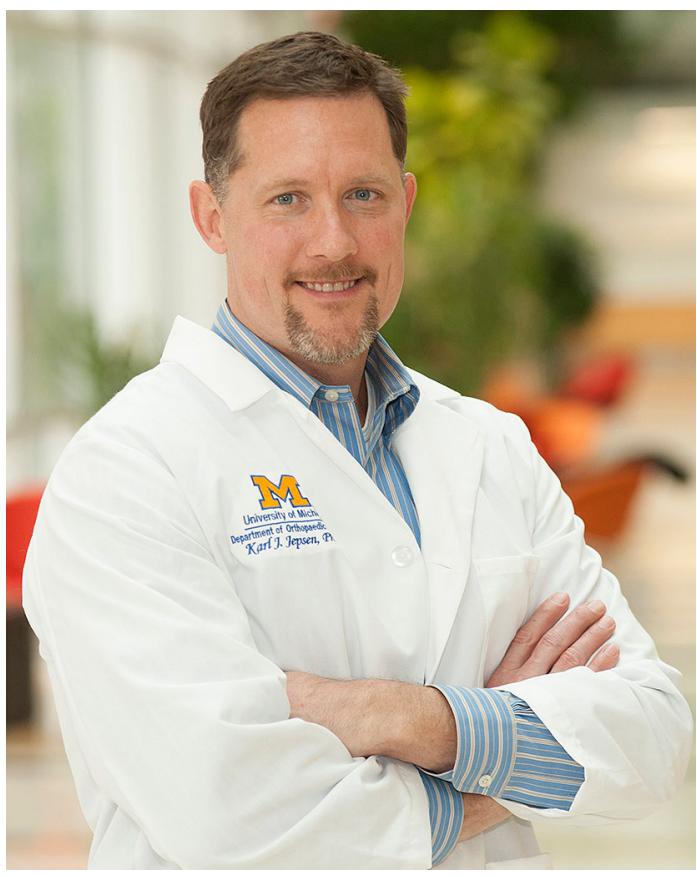

Karl J. Jepsen PhD

J. H. Healey MD

Orthopaedic Surgery Service, Memorial Sloan-Kettering Cancer Center, New York, NY, USA

L. L. Tosi MD ( $\varangle)$

Division of Orthopaedic Surgery and Sports Medicine, Children's National Health System, 111 Michigan Ave NW, Washington, DC 20010, USA

e-mail: 1tosi@cnmc.org species. In 1993, Congress passed the NIH Revitalization Act, mandating that women and minorities be included in all clinical research, and requiring that Phase III clinical trials be analyzed by sex. Subsequently, a 2001 Institute of Medicine (IOM) report [1] unequivocally stated that "every cell has a sex," and research should take 


\section{Symposium: Sex Differences in Musculoskeletal Disease and Science}

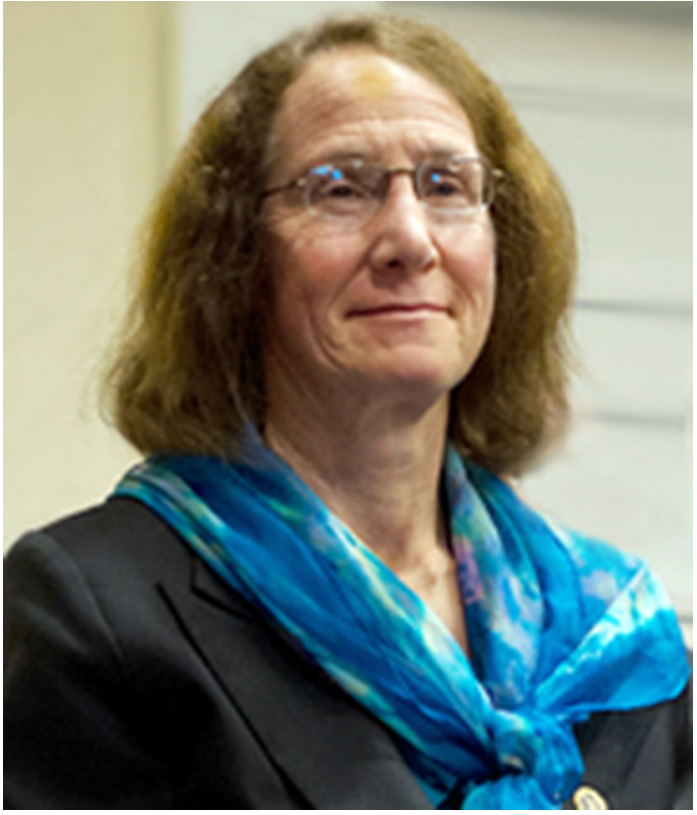

Nancy E. Lane MD

sex differences into consideration from "womb to tomb."

The differences between men and women are important to-but incompletely characterized in-musculoskeletal research. Differing incidence and severity of musculoskeletal diseases, conditions, and sports injuries between men and women are the result of inherent anatomic, biomechanical, hormonal, and cellular/molecular differences. While traumatic injuries are more common in men than in women, women are disproportionately disabled by musculoskeletal conditions, and have a higher incidence of spinal deformities and ACL injuries during adolescence, stress fractures during adulthood and osteoarthritis, rotator cuff tears, and osteoporotic fragility fractures during aging compared to men. Differences in the mechanisms of injury, pain sensation, drug handling, and healing response have biologic bases, but how they are influenced by sex or gender is not well understood. Differences can also be found between the two genders when comparing responses to surgery, anesthesia, medications, and rehabilitation [2].

In an effort to underscore and expand the findings of the IOM report, as well as to explore how sex- and gender-driven biologic and physiologic characteristics drive differences in musculoskeletal health, the American Academy of Orthopaedic Surgeons (AAOS), the National Institute of Arthritis and Musculoskeletal and Skin Diseases of the National Institutes of Health (NIH/NIAMS), and the NIH Office of Research in Women's Health (NIH/ORWH) cosponsored a 2004 research symposium: "Does Sex Matter in Musculoskeletal Health? The Influence of Sex and Gender on Musculoskeletal Health" [3]. The workshop underscored the importance of sex differences in all tissues of the musculoskeletal system. The workshop summary emphasized the need for greater awareness of sex differences in the design of in vitro and preclinical animal studies, and the need for appropriately powered studies to be able to identify sex differences in the human population.

Although this first workshop influenced the thinking of many basic scientists and clinicians, progress in 


\section{Symposium: Sex Differences in Musculoskeletal Disease and Science}

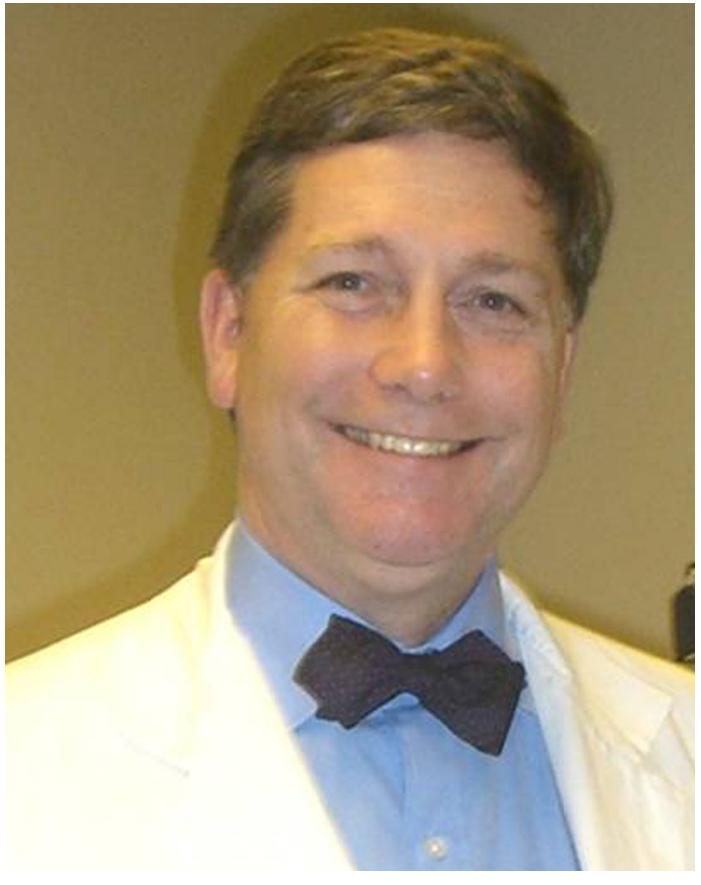

John H. Healey MD

translating the 2004 findings into improved clinical practice has been disappointing, especially how these findings impact different stages of the life cycle (a particularly important issue for women). More research needs to be done to define clearly how and when men and women differ across the lifespan, and when these differences are clinically important. To reignite interest and raise awareness of new discoveries on the importance of sex differences in the musculoskeletal community, the original workshop leadership team partnered with the Association of Bone and Joint Surgeons ${ }^{\mathbb{R}}$ (ABJS, the parent society of Clinical Orthopaedics and Related Research $\left.{ }^{\mathbb{R}}\left[\operatorname{CORR}^{\circledR}\right]\right)$, the AAOS, NIH/NIAMS, the Orthopaedic Research Society, the Center for Musculoskeletal Health at the University of California, Davis, and the Society for
Women's Health Research, as well as the current and past chairs of the AAOS Women's Health Issues Advisory Board, to develop the current symposium, "Musculoskeletal Sex Differences Across the Lifespan," held July 31 to August 1, 2014 in Rosemont, IL, USA. The purpose of this second workshop was to bring together clinicians, researchers, representatives of the NIH and the FDA, members of industry, young investigators, and leaders of major research centers to review recent advances in our understanding of how sex and gender differences influence musculoskeletal health and to concentrate on the fact that sex differences may actually change in time.

The symposium focused on lifespan, exploring the state of knowledge of musculoskeletal sex and gender differences during growth, adolescence, adulthood, and aging. The scientific sessions inspired cross-disciplinary interaction, a major mission of the workshop. The discussion focused on factors during early growth that impact the development of musculoskeletal conditions during aging. The interaction between growth and aging, although understudied, is likely to be critical, as new research is showing. Many diseases affecting 


\section{Symposium: Sex Differences in Musculoskeletal Disease and Science}

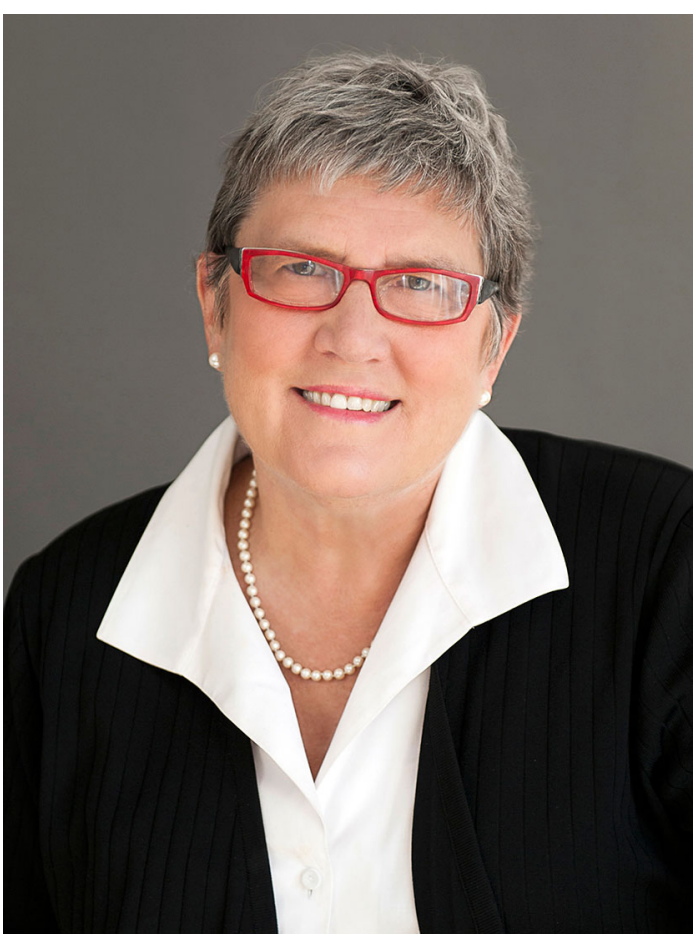

Laura L. Tosi MD

older patients (including osteoporosis, age-related osteoarthritis, and rotator cuff tears) are often thought to be natural sequelae of aging, yet many of the contributing factors to these musculoskeletal conditions may originate earlier in life, when individuals are generally "healthy." For example, osteoporosis appears, at least in part, to be a disease of childhood and adolescence, and various anatomical factors defined during growth may increase soft tissue pathology.

In this volume of $C O R R^{\circledR}$, we present a collection of papers submitted by workshop participants, particularly young investigators, which underscore the critical importance of both sex- and gender-related changes during the course of normal aging in determining musculoskeletal health. However, these manuscripts also acknowledge that the exact mechanisms involved in these differences have not yet been well described or disseminated to clinical audiences, nor have they been correlated with clinical observations. Above all, the collection underscores the key message found in the recent editorial written by the $C O R R^{\circledR}$ editorial board: Recognition of sex and gender differences in the scientific analysis of musculoskeletal disorders is "not just a semantic issue. It is a health issue" [2].

Defining where men and women differ in terms of musculoskeletal diseases will provide guidance to basic scientists in focusing research efforts on clinically important problems. An approach tailored to the biological needs of women and men separately is urgently needed. Moreover, consideration of age-related changes is critical, as many of the conditions that affect musculoskeletal tissues are age dependent.

A bifurcated approach should benefit both genders, but especially women, who live longer than men, and demographic trends predict that the majority of senior citizens will be women. Chronic conditions found in this population frequently involve one or more musculoskeletal problems. As our armamentarium of treatment options increases, recognition of sex- 


\section{Symposium: Sex Differences in Musculoskeletal Disease and Science}

and gender-related differences will allow healthcare providers to address each patient based on his or her personal biological needs.

The co-chairs would like to thank the many individuals who contributed to the success of the research symposium and produced this collection of papers, especially Barbara Boyan $\mathrm{PhD}$, Adele Boskey PhD, Mary O'Connor $\mathrm{MD}$, Elizabeth Arendt MD, and Laura Gehrig MD for their service on the workshop planning committee. We are indebted to the AAOS, and particularly Erin Ransford, AAOS Manager, Research Advocacy, for providing the excellent administrative and organizational support essential to an effective meeting. Finally, we are deeply grateful to Dr. Seth Leopold, Editor-in-Chief of $C O R R^{\circledR}$, and the ABJS for choosing this project as a workshop topic, and providing the substantial financial and publishing support which allowed us to create a single body of work that so compellingly underscores many of the directions that need to be pursued in order to advance our understanding and treatment of musculoskeletal conditions in both men and women across the lifespan.

\section{References}

1. Institute of Medicine (US) Committee on Understanding the Biology of Sex and Gender Differences. Exploring the Biological Contributions to Human Health: Does Sex Matter? Washington, DC: National Academy Press; 2001.

2. Leopold SS, Beadling L, Dobbs MB, Gebhardt MC, Lotke PA, Manner PA, Rimnac CM, Wongworawat MD. Fairness to all: Gender and sex in scientific reporting. Clin Orthop Relat Res. 2014;472:391-392.

3. Tosi LL, Boyan BD, Boskey AL. Does sex matter in musculoskeletal health? J Bone Joint Surg Am. 2005; 87-A:1631-1647. 